\title{
First-Principles Study of Electronic Structure and Optical Properties of Tetragonal $\mathrm{PbMoO}_{4}$
}

\author{
Qi-Jun Liu, Zheng-Tang Liu, Li-Ping Feng, and Hao Tian \\ State Key Laboratory of Solidification Processing, School of Materials Science and Engineering, Northwestern Polytechnical University, \\ Xi'an, Shaanxi 710072, China \\ Correspondence should be addressed to Qi-Jun Liu, dianerliu@yahoo.com.cn
}

Received 24 July 2011; Accepted 24 August 2011

Academic Editors: S. Bud'ko and A. Zagoskin

Copyright ( 2011 Qi-Jun Liu et al. This is an open access article distributed under the Creative Commons Attribution License, which permits unrestricted use, distribution, and reproduction in any medium, provided the original work is properly cited.

Using the plane-wave ultrasoft pseudopotential technique based on the first-principles density functional theory (DFT), we have studied the structural, electronic, chemical bonding, and optical properties of tetragonal $\mathrm{PbMoO}_{4}$. The obtained structural parameters are in good agreement with experiments. Band structure, density of states, and chemical bonding are calculated and shown. It is found that tetragonal $\mathrm{PbMoO}_{4}$ is an indirect band gap. The dielectric function, refractive index, extinction coefficient, reflectivity, absorption coefficient, loss function, and conductivity function are calculated for radiation up to $20 \mathrm{eV}$.

\section{Introduction}

$\mathrm{PbMoO}_{4}$ has been the subject of great research interest both experimentally [1-8] and theoretically [9-13] due to its wide applications such as acousto-optic light deflectors, modulators, adjustable filters, surface acoustic wave devices, ionic conductors, and low-temperature scintillators, and its superior properties such as high acousto-optic light figure of merit, low optical loss in the region 420-3900 nm, and good mechanical impedance for acoustic matching ([14-17] and the references therein).

The crystal structure of tetragonal $\mathrm{PbMoO}_{4}$ belongs to the space group $I 4_{1} / a$ and the local symmetry $C_{4 h}^{6}$. The dielectric constants [18], polarized reflectivity spectra [19], high-pressure Raman spectrum, and electrical properties $[1,7,14]$ have been reported, which show that there is an anisotropy of optical properties and a transition from crystal to amorphous phase with increasing pressure. The framework of a fully relativistic self-consistent Dirac-Slater theory with a numerically discrete variational (DV-X $\alpha$ ) method $[9,13,19]$, the ultrasoft pseudopotentials of generalized gradient approximations (GGAs) based on the density function theory (DFT) with CASTEP code $[10,12]$, the linearized-augmented-plane-wave method with WIEN97 code [11], and so forth have been used to study F type color centers, optical properties, electronic band structure, and so forth. Although these studied cases of $\mathrm{PbMoO}_{4}$ are well presented, it is not clear how electron transitions to influence optical properties is. Additionally, the chemical bonding of $\mathrm{PbMoO}_{4}$ should be explained.

Hence, we study the structural parameters, electronic structure, chemical bonding, and optical properties of tetragonal $\mathrm{PbMoO}_{4}$ using the plane-wave ultrasoft pseudopotential technique based on the first-principles density functional theory. The rest of the work is organized as follows. In Section 2, we give a short description of the methods used in this paper. The results and discussion are shown in Section 3. We present our findings and give a brief summary in Section 4.

\section{Computational Methodology}

Density functional theory calculations are performed with plane-wave ultrasoft pseudopotential using the generalized gradient approximation (GGA) with the Perdew-Wang 1991 (PW91) functional [20] as implemented in the CASTEP code [21]. The ionic cores are represented by ultrasoft pseudopotentials for $\mathrm{Mo}, \mathrm{Pb}$, and $\mathrm{O}$ atoms. The $\mathrm{Mo} 4 \mathrm{~s}^{2} 4 \mathrm{p}^{6} 4 \mathrm{~d}^{5} 5 \mathrm{~s}^{1}, \mathrm{~Pb}$ $5 \mathrm{~d}^{10} 6 \mathrm{~s}^{2} 6 \mathrm{p}^{2}$, and $\mathrm{O} 2 \mathrm{~s}^{2} 2 \mathrm{p}^{4}$ electrons are explicitly treated as valence electrons. The plane-wave cutoff energy is $380 \mathrm{eV}$, 


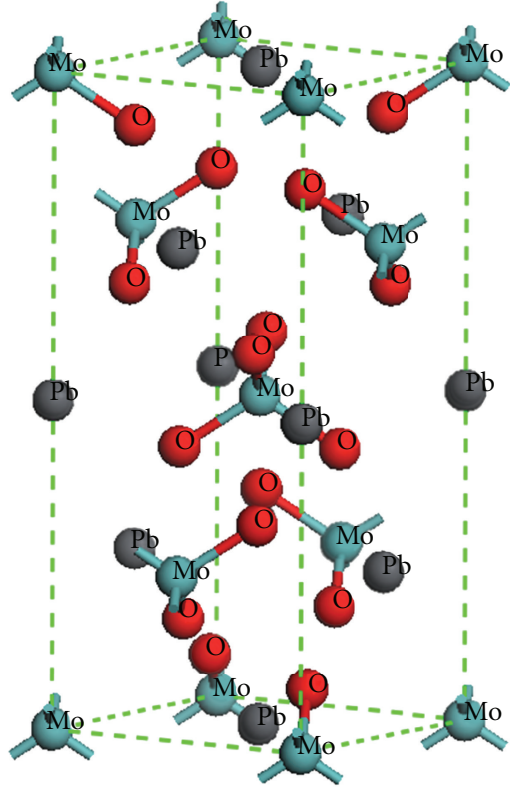

Figure 1: The crystal structure of tetragonal $\mathrm{PbMoO}_{4}$.

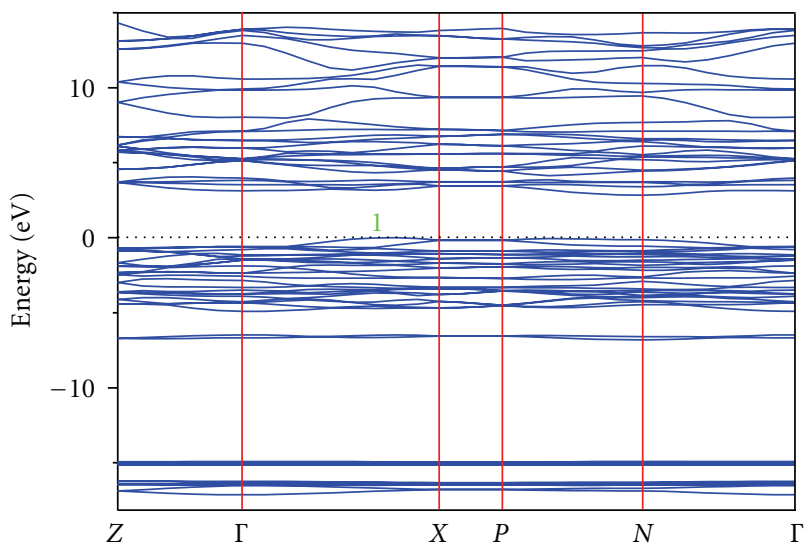

Figure 2: Band structure of tetragonal $\mathrm{PbMoO}_{4}$ along with the high-symmetry points of the Brillouin zone.

and the Brillouin zone integration is performed over the $5 \times 5 \times 6$ grid sizes using the Monkhorst-Pack method for tetragonal structure optimization. This set of parameters assures the maximum force of $0.01 \mathrm{eV} / \AA$, the maximum stress of $0.02 \mathrm{GPa}$, and the maximum displacement of $5.0 \times$ $10^{-4} \AA$.

\section{Results and Discussion}

3.1. Geometry and Structure Optimization. The crystal structure of tetragonal $\mathrm{PbMoO}_{4}$ is shown in Figure 1. The optimized values of $a$ and $c$ for tetragonal $\mathrm{PbMoO}_{4}$ are listed in Table 1. The obtained structural parameters are in good agreement with the previous experimental data $[2,11,16]$. Of course, it can be seen that the GGA leads to overestimating our calculated data compared with experiments.

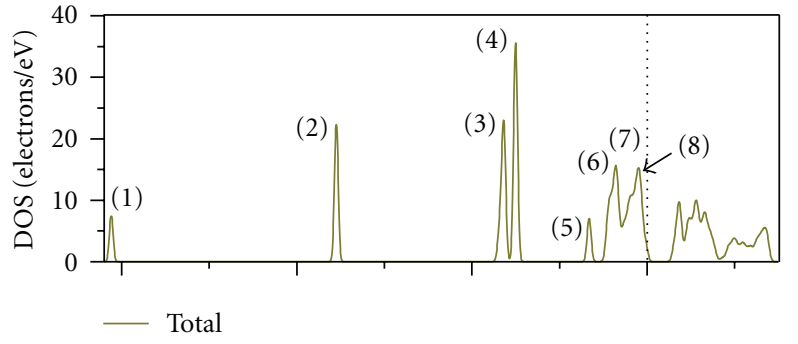

(a)

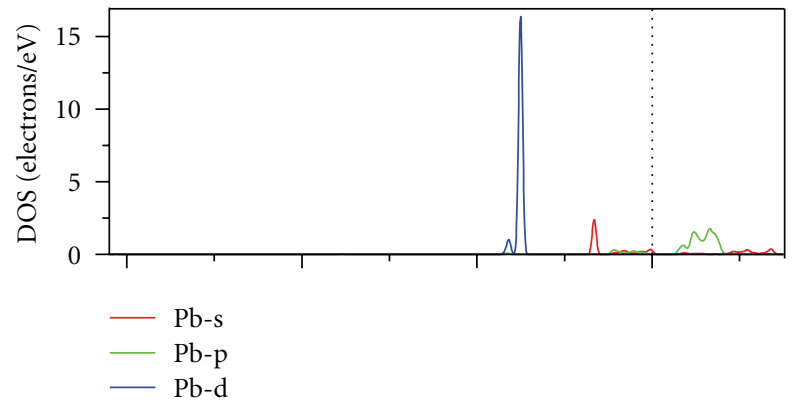

(b)

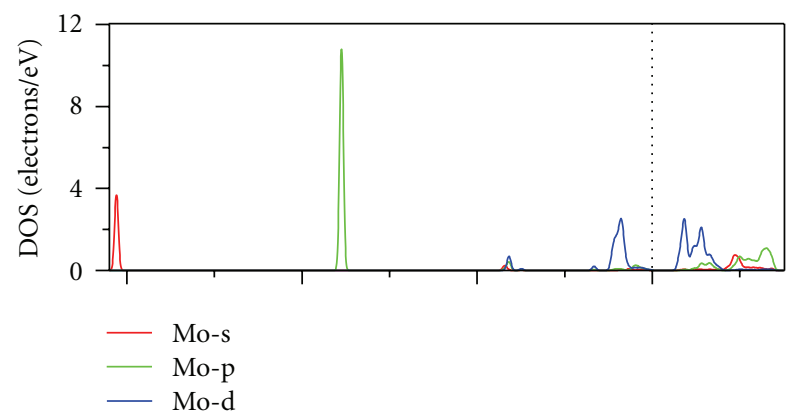

(c)

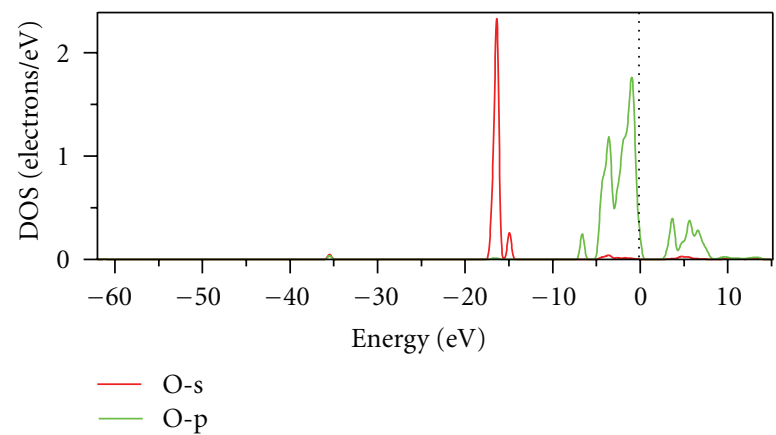

(d)

FIgUre 3: Total and partial density of states of tetragonal $\mathrm{PbMoO}_{4}$.

3.2. Electronic Properties. The calculations of the electronic band structure along the symmetry lines of the Brillouin zone, the total and the partial density of states (DOSs and PDOSs) are shown in Figures 2 and 3. The top of the valence band is taken as the zero of energy. In this compound, the valence band maximum (VBM) is located at 1 point (the valence band maximum of tetragonal $\mathrm{PbMoO}_{4}$ is not at high-symmetry point, but at the defined 1 point between 


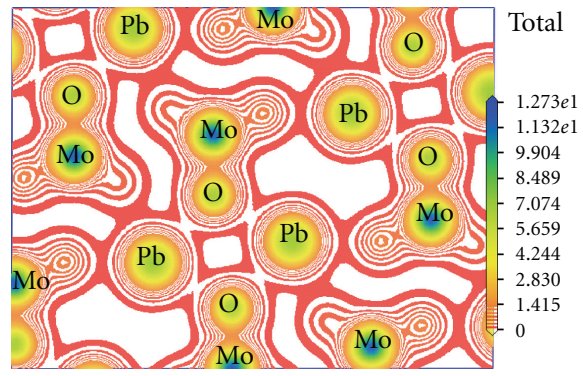

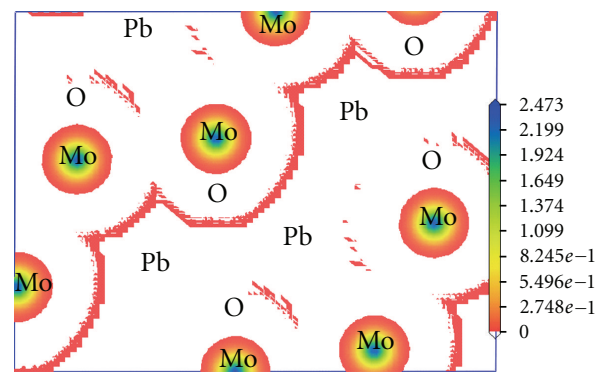

(1)

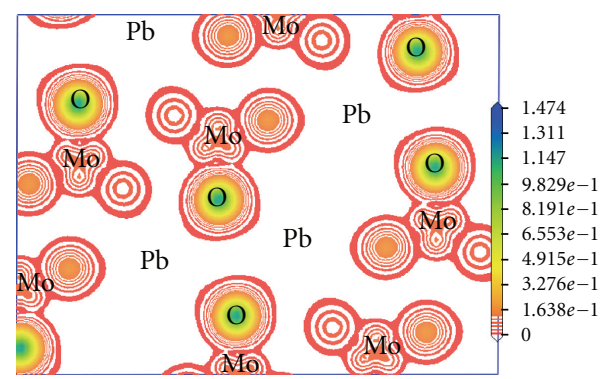

(3)

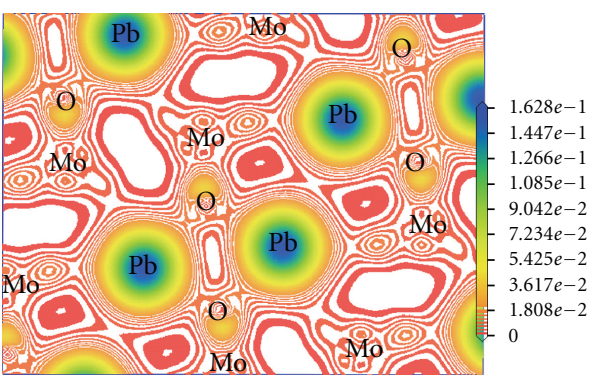

(5)

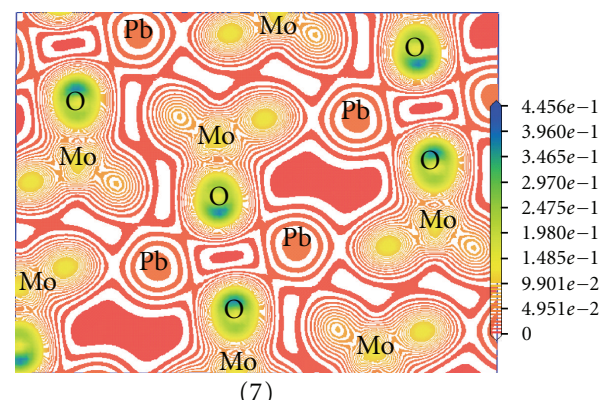

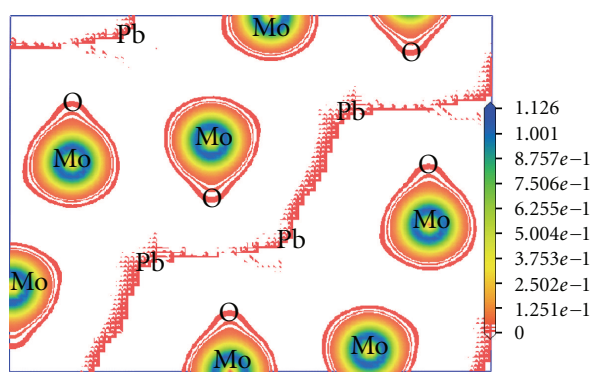

(2)

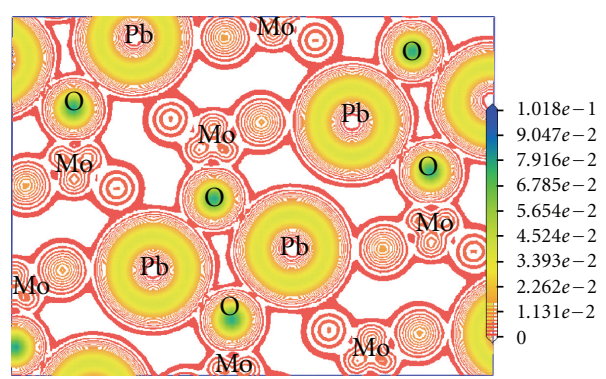

(4)

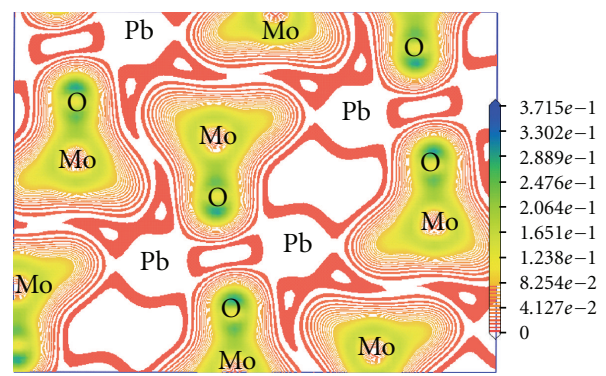

(6)

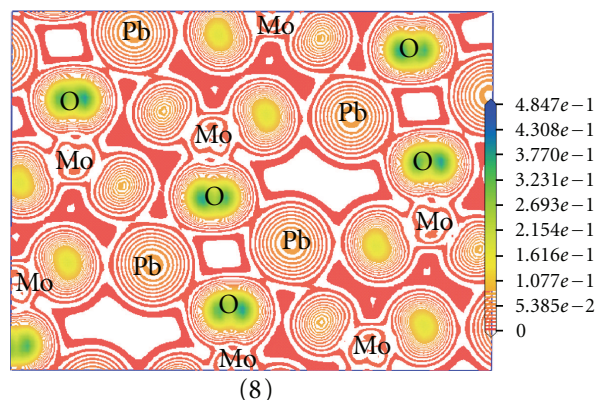

FIgURE 4: Total charge densities of (112) plane and charge densities located (1)-(8) in the (112) plane of tetragonal $\mathrm{PbMoO}_{4}$. 


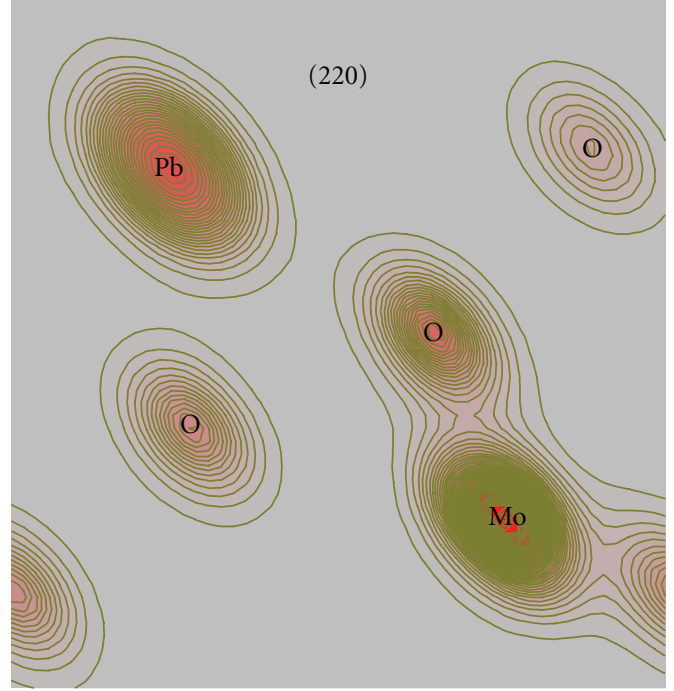

Figure 5: Charge densities in the (220) plane of tetragonal $\mathrm{PbMoO}_{4}$.

TABLE 1: Calculated equilibrium lattice parameters $a, c$ (in $\AA$ ), and c/a compared with available experimental data $[2,11,16]$ for tetragonal $\mathrm{PbMoO}_{4}$.

\begin{tabular}{lcccc}
\hline \multicolumn{5}{c}{ This work } \\
Castep & & & \\
& GGA & Expt. [11] & Expt. [16] & Expt. [2] \\
& (PW91) & & & \\
\hline$a$ & 5.5173 & $5.424-5.4360$ & 5.433 & $5.418-5.464$ \\
$c$ & 12.3305 & $12.076-12.1107$ & 12.110 & $12.065-12.088$ \\
$c / a$ & 2.2349 & $2.226-2.229$ & - & - \\
\hline
\end{tabular}

$X$ and $\Gamma$ ), whereas the conduction band minimum (CBM) is located at $N$ point, resulting in indirect band gap of $2.838 \mathrm{eV}$. This value is in good agreement with the previous calculated data $2.59 \mathrm{eV}$ [11] and $2.8 \mathrm{eV}$ [12]. However, these results are all smaller than the experimental values of $2.94-4.7 \mathrm{eV}$ [2] due to the well-known underestimation of conduction band energy in DFT calculations [22].

In order to further elucidate the nature of the electronic band structure, we have calculated and explained the DOSs and PDOSs. From the PDOSs, we can identify the angular momentum character of the different structures. Structure (1) is mainly due to Mo-4s electrons, structure (2) due to Mo-4p electrons, structure (3) due to O-2s electrons, structure (4) due to $\mathrm{Pb}-5 \mathrm{~d}$ electrons, structure (5) due to $\mathrm{Pb}-6$ s electrons, structures (6) and (7) due to O-2p electrons with hybridization of Mo-4d electrons, and structure (8) due to $\mathrm{O}-2 \mathrm{p}$ electrons. The conduction bands are composed of Mo-4d and show the hybridization with $\mathrm{O}-2 \mathrm{p}$, as well as the hybridization between $\mathrm{Pb}-6 \mathrm{p}$ and $\mathrm{O}-2 \mathrm{p}$.

To understand the chemical bonding of this material, we have poltted the charge density of (112) plane corresponding to the (1)-(8) located at Figure 3 in Figure 4. The polt labeled (1) shows the isolated Mo- $4 \mathrm{~s}$, (2) the weak hybridization $\sigma$ bonding between Mo-4p and O-2s, (3) the

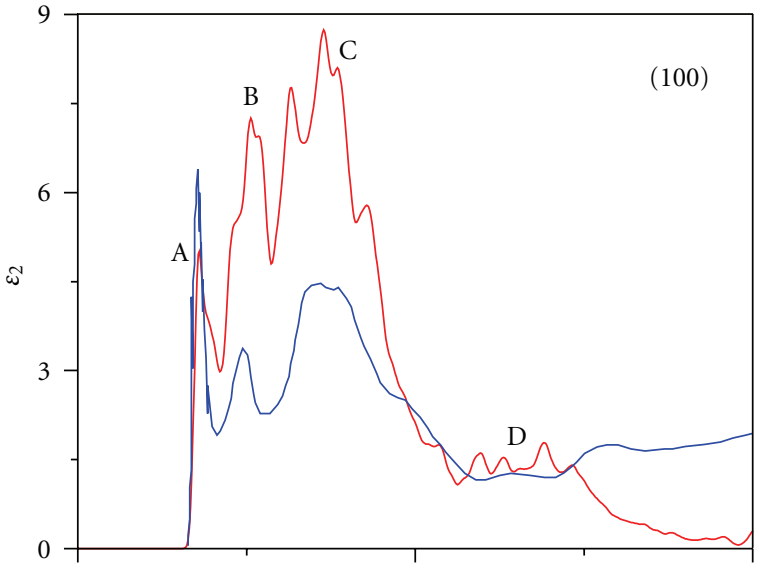

(a)

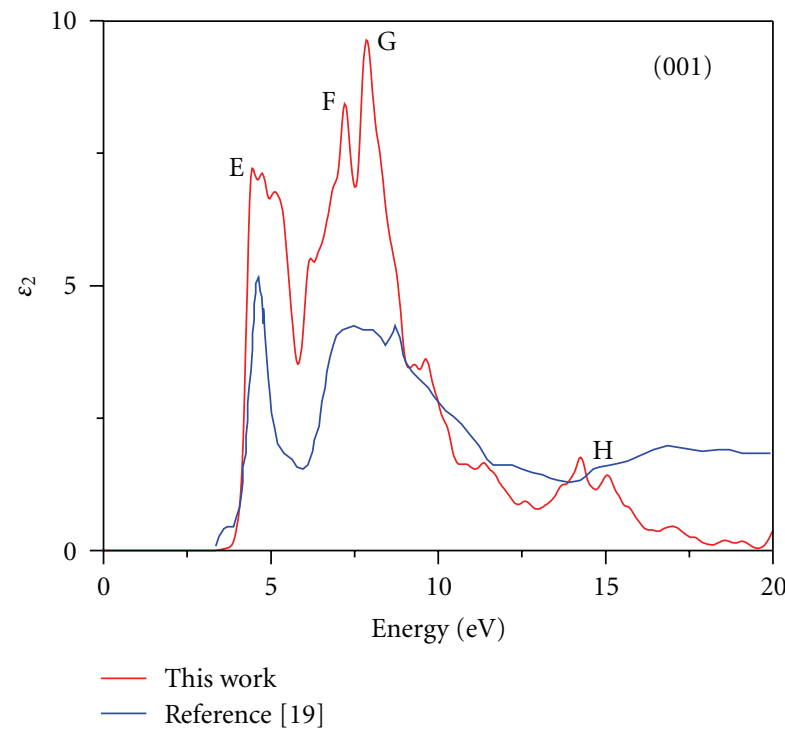

(b)

Figure 6: Calculated imaginary parts of complex dielectric function of tetragonal $\mathrm{PbMoO}_{4}$ as well as calculated results from experimental data [19].

weak hybridization $\sigma$ bonding between $\mathrm{O}-2 \mathrm{~s}$ and Mo-4d, (4) the weak hybridization $\sigma$ bonding between Pb-5d and $\mathrm{O}-2 \mathrm{~s},(5)$ the weak hybridization $\sigma$ bonding between $\mathrm{Pb}-6 \mathrm{~s}$ and O-2p, (6) and (7) the hybridization $\sigma$ and $\pi$ bonding between O-2p and Mo-4d, and (8) the nonbonding $\mathrm{O}-2 \mathrm{P}_{\pi}$. Hence, we can conclude that the bonding between Mo and $\mathrm{O}$ is mainly covalent and the bonding between $\mathrm{Pb}$ and $\mathrm{O}$ is mainly ionic. Additionally, the charge density of (220) plane and the results of population analysis have been shown in Figure 5 and Table 2, which are in good agreement with our analysis of chemical bonding.

3.3. Optical Properties. We need to calculate two dielectric tensor components to completely characterize the linear optical properties due to the tetragonal symmetry of $\mathrm{PbMoO}_{4}$. The imaginary and the real parts of the dielectric function are 


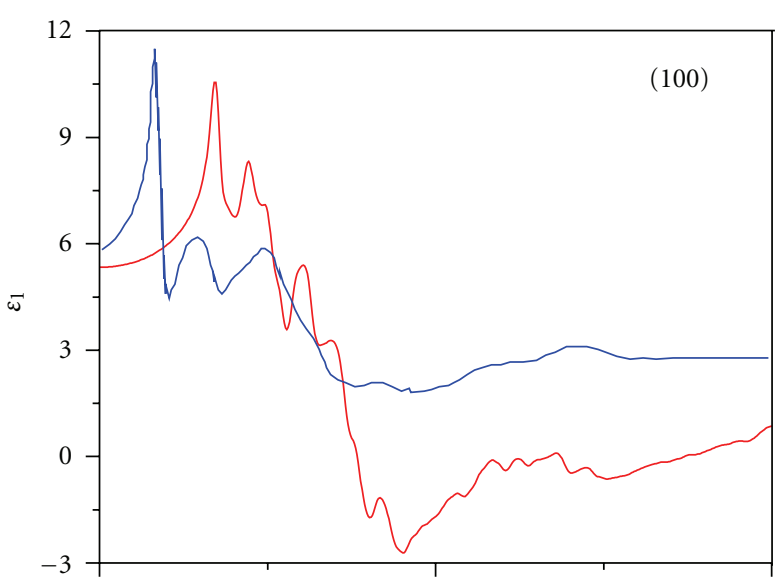

(a)

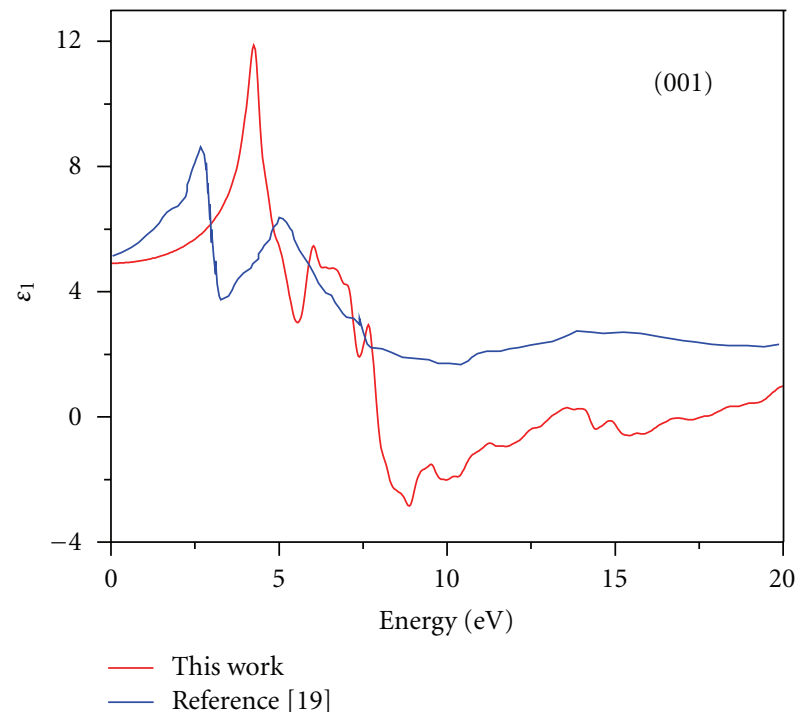

(b)

Figure 7: Calculated real parts of complex dielectric function of tetragonal $\mathrm{PbMoO}_{4}$ as well as calculated results from experimental data [19].

TABLE 2: Calculated results of population analysis.

\begin{tabular}{lccccc}
\hline Atom & $\mathrm{s}$ & $\mathrm{p}$ & $\mathrm{d}$ & Total & Charge $(\mathrm{e})$ \\
\hline $\mathrm{O}$ & 1.89 & 4.78 & 0 & 6.68 & -0.68 \\
$\mathrm{Mo}$ & 2.24 & 6.51 & 4.01 & 12.76 & 1.24 \\
$\mathrm{~Pb}$ & 1.71 & 0.81 & 10 & 12.52 & 1.48 \\
\hline
\end{tabular}

calculated using (1) [23-25]:

$$
\begin{gathered}
\varepsilon_{2}(\omega)=\left(\frac{4 \pi^{2} e^{2}}{m^{2} \omega^{2}}\right) \sum_{i, j} \int\langle i|M| j\rangle^{2} f_{i}\left(1-f_{i}\right) \delta\left(E_{f}-E_{i}-\omega\right) d^{3} k, \\
\varepsilon_{1}(\omega)=1+\frac{2}{\pi} P \int_{0}^{\infty} \frac{\omega^{\prime} \varepsilon_{2}\left(\omega^{\prime}\right) d \omega^{\prime}}{\left(\omega^{\prime 2}-\omega^{2}\right)}
\end{gathered}
$$

as well as the scissors operator approximation $[25,26]$ due to underestimating the energies of excitation with the den-

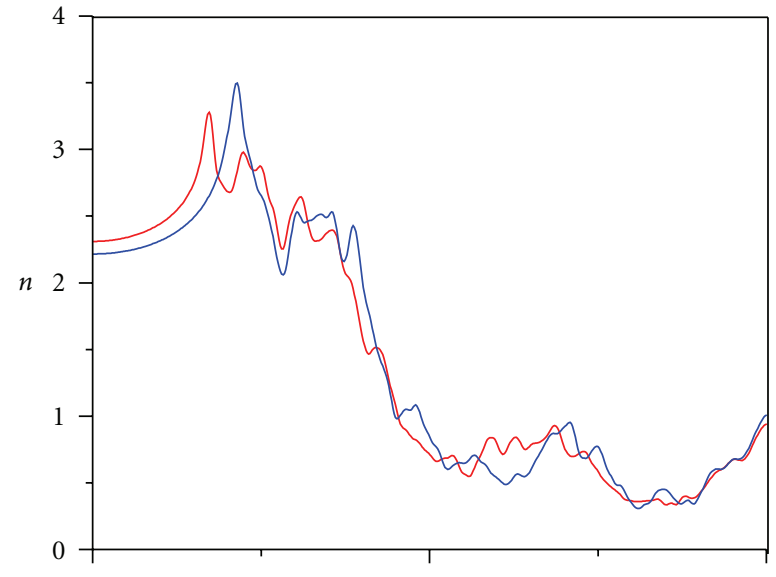

(a)

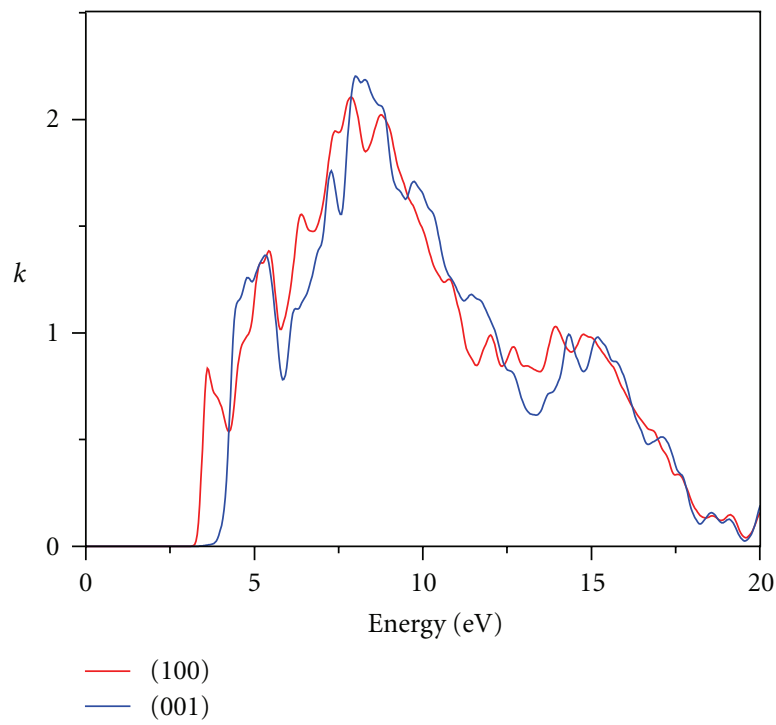

(b)

Figure 8: Calculated refractive index and extinction coefficient of tetragonal $\mathrm{PbMoO}_{4}$ from (100) and (001).

sity functional calculations. The good agreement with experiments are obtained for the optical properties like $\mathrm{TiO}_{2}[26]$, $\mathrm{SrHfO}_{3}$ [27], $\mathrm{SrZrO}_{3}$ [28], and $\mathrm{HfO}_{2}$ [29] using the scissors operator.

Figures 6 and 7 display the imaginary and the real parts of the dielectric function from (100) and (001) along with the calculated results from experimental data [19] for a radiation up to $20 \mathrm{eV}$. We can see that our results are consistent with the previous work [19]. The discrepancy between our results and the experiment [19] may be due to the different-temperature condition ( $0, \mathrm{~K}$ in our paper and $6, \mathrm{~K}$ in [19]). The imaginary parts exhibit four structures A-D of (100) and E-H of (001). Structures A and $\mathrm{E}$ originate mainly from transitions of $\mathrm{O}-$ $2 \mathrm{p}_{\pi}$ into the conduction bands, and structures $\mathrm{B}$ and $\mathrm{F}$ from transitions of hybridization $\pi$ bonding between $\mathrm{O}-2 \mathrm{p}$ and Mo-4d into the conduction bands, and structures $\mathrm{C}$ and $\mathrm{G}$ from transitions of hybridization $\sigma$ bonding between $\mathrm{O}-2 \mathrm{p}$ and Mo- $4 \mathrm{~d}$ into the conduction bands, and structures $\mathrm{D}$ and 


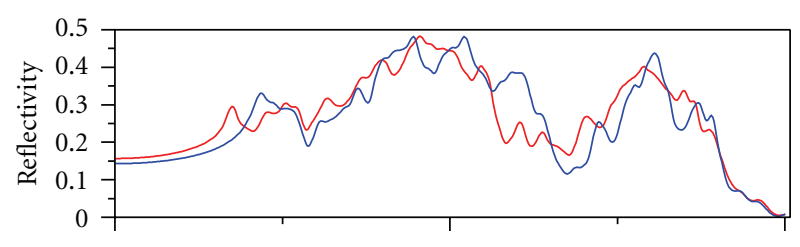

(a)

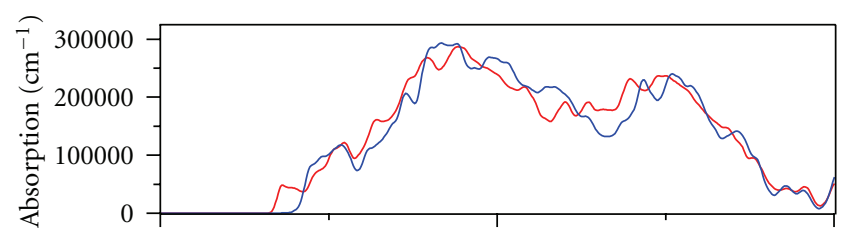

(b)

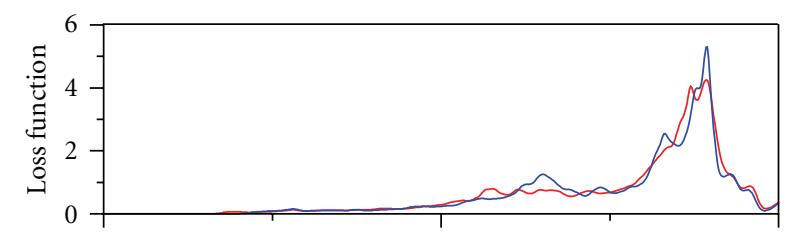

(c)

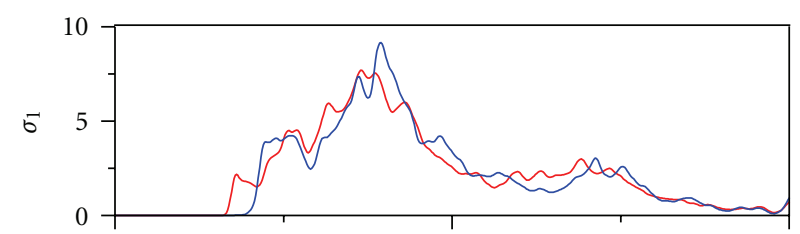

(d)

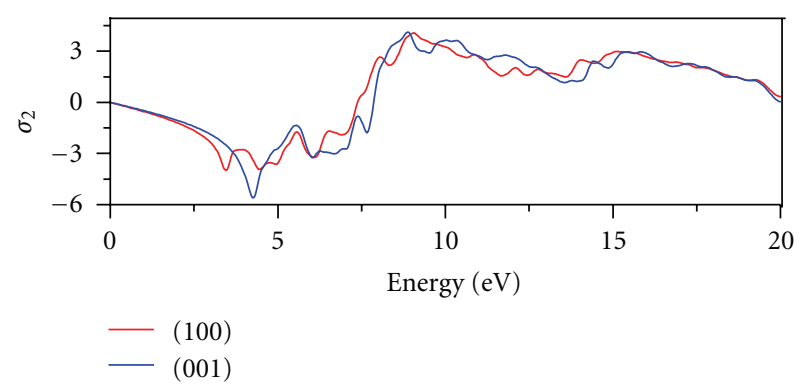

(e)

FIGURE 9: Calculated reflectivity, absorption coefficient, loss function and complex conductivity function of tetragonal $\mathrm{PbMoO}_{4}$ from (100) and (001).

$\mathrm{H}$ from transitions of $\mathrm{Pb}-6 \mathrm{~s}$ into the conduction bands. The calculated static dielectric constants are 5.337 and 4.910 from (100) and (001).

The refractive index and the extinction coefficient are displayed in Figure 8. The static refractive index is found to have the values 2.310 and 2.216 from polarization vectors (100) and (001), which are in agreement with experimental data 2.28 and 2.40 [18] from (100) and (001). Figure 9 shows the calculated results on the reflectivity, absorption coefficient, loss function, and complex conductivity function from polarization vectors (100) and (001). We hope the calculated values can help to offer a theoretical basis for the experiment and application of tetragonal $\mathrm{PbMoO}_{4}$.

\section{Conclusions}

The paper reports detailed investigations on the structural, electronic, chemical bonding, and optical properties of tetragonal $\mathrm{PbMoO}_{4}$ using the plane-wave ultrasoft pseudopotential technique based on the first-principles density-functional theory (DFT). The calculated equilibrium lattice parameters are in agreement with experiments. Our calculated results of the band structure and DOSs show that this compound is an indirect band gap of $2.838 \mathrm{eV}$. The charge densities and population analysis are obtained and analyzed, which show that Mo and $\mathrm{O}$ are mainly covalent, whereas $\mathrm{Pb}$ and $\mathrm{O}$ are mainly ionic. The complex dielectric function has been shown, and the peaks position distributions of imaginary parts of complex dielectric function have been explained, which show electron transitions in the electronic bands.

\section{Acknowledgments}

This work was financially supported by the National Natural Science Foundation of China (Contract no. 50902110), the Doctorate Foundation of Northwestern Polytechnical University (Contract no. cx201005), the 111 Project (Contract no. B08040), and the Research Fund of the State Key Laboratory of Solidification Processing (NWPU), China (Contract no. 58-TZ-2011).

\section{References}

[1] C.-L. Yu, Q.-J. Yu, C.-X. Gao et al., "In-situ high pressure Raman spectrum and electrical property of $\mathrm{PbMoO}_{4}$," Chinese Physics Letters, vol. 24, no. 8, article 014, pp. 2204-2207, 2007.

[2] J. C. Sezancoski, M. D.R. Bomio, L. S. Cavalcante et al., "Morphology and blue photoluminescence emission of $\mathrm{PbMoO}_{4}$ processed in conventional hydrothermal," Journal of Physical Chemistry C, vol. 113, no. 14, pp. 5812-5822, 2009.

[3] J. A. Groenink and H. Binsma, "Electrical conductivity and defect chemistry of $\mathrm{PbMoO}_{4}$ and $\mathrm{PbWO}_{4}$," Journal of Solid State Chemistry, vol. 29, no. 2, pp. 227-236, 1979.

[4] S. C. Sabharwal, Sangeeta, and D. G. Desai, "Investigations of single crystal growth of $\mathrm{PbMoO}_{4}$," Crystal Growth and Design, vol. 6, no. 1, pp. 58-62, 2006.

[5] T. T. Zhou, H. L. Hu, and S. Q. Sun, "Studies of infrared antireflection coating on $\mathrm{PbMoO}_{4}$ single crystal," Vacuum Science and Technology, vol. 22, p. 392, 2002.

[6] J. C. Wang, C. X. Liu, and Y. C. Ge, "Research on growth and optical properties of $\mathrm{PbMoO}_{4}$ crystal," Journal of Synthetic Crystals, vol. 24, p. 238, 1995.

[7] C.-L. Yu, Q.-J. Yu, C.-X. Gao et al., "Investigation of in-situ Raman spectrum and electrical conductivity of $\mathrm{PbMoO}_{4}$ at high pressure," Chinese Journal of High Pressure Physics, vol. 21, no. 3, pp. 259-263, 2007.

[8] W.-P. Zhou, S.-M. Wan, X. Zhang et al., "Study of growth units and the growth habit of $\mathrm{PbMoO}_{4}$ crystal using high temperature Raman spectra," Acta Physica Sinica, vol. 57, no. 11, pp. 7305-7309, 2008.

[9] J. Chen, Q. Zhang, T. Liu, Z. Shao, and C. Pu, "Electronic structures of $\mathrm{PbMoO}_{4}$ crystal with F-type color centers," Chinese Journal of Computational Physics, vol. 25, no. 2, pp. 213-217, 2008. 
[10] Z. C. Guo, H. N. Dong, B. Deng, and D. F. Li, "Firstprinciples study of the optical properties for the $\mathrm{PbMoO}_{4}$ crystal," Material Review, vol. 24, p. 237, 2010.

[11] Y. Zhang, N. A. W. Holzwarth, and R. T. Williams, "Electronic band structures of the scheelite materials $\mathrm{CaMoO}_{4}, \mathrm{CaWO}_{4}$, $\mathrm{PbMoO}_{4}$, and $\mathrm{PbWO}_{4}$," Physical Review B, vol. 57, no. 20, pp. 12738-12750, 1998.

[12] J. Chen, T. Liu, D. Cao, and G. Zhao, "First-principles study of the electronic structures and absorption spectra for the $\mathrm{PbMoO}_{4}$ crystal with lead vacancy," Physica Status Solidi B, vol. 245, no. 6, pp. 1152-1155, 2008.

[13] J. Y. Chen, Q. R. Zhang, T. Y. Liu, and Z. Y. Shao, "Firstprinciples study of color centers in $\mathrm{PbMoO}_{4}$ crystals," Physica B: Condensed Matter, vol. 403, no. 4, pp. 555-558, 2008.

[14] C. L. Yu, Q. J. Yu, C. X. Gao et al., "Structural and electrical properties of $\mathrm{PbMoO}_{4}$ under high pressure," Journal of Physics Condensed Matter, vol. 19, no. 42, 2007.

[15] H. C. Zeng, "Correlation of $\mathrm{PbMoO}_{4}$ crystal imperfections to Czochralski growth process," Journal of Crystal Growth, vol. 171, no. 1-2, pp. 136-145, 1997.

[16] N. Senguttuvan, S. M. Babu, and R. Dhanasekaran, "Some aspects on the growth of lead molybdate single crystals and their characterization," Materials Chemistry and Physics, vol. 49, no. 2, pp. 120-123, 1997.

[17] M. Tyagi, S. G. Singh, A. K. Singh, and S. C. Gadkari, "Understanding colorations in $\mathrm{PbMoO}_{4}$ crystals through stoichiometric variations and annealing studies," Physica Status Solidi A, vol. 207, no. 8, pp. 1802-1806, 2010.

[18] W. S. Brower and P. H. Fang, "Dielectric constants of $\mathrm{PbMoO}_{4}$ and $\mathrm{CaMoO}_{4}$," Physical Review, vol. 149, no. 2, p. 646, 1966.

[19] M. Fujita, M. Itoh, H. Mitani, S. Sangeeta, and M. Tyagi, "Exciton transition and electronic structure of $\mathrm{PbMoO}_{4}$ crystals studied by polarized light," Physica Status Solidi B, vol. 247, no. 2, pp. 405-410, 2010.

[20] J. P. Perdew, J. A. Chevary, S. H. Vosko et al., "Atoms, molecules, solids, and surfaces: applications of the generalized gradient approximation for exchange and correlation," Physical Review B, vol. 46, no. 11, pp. 6671-6687, 1992.

[21] M. D. Segall, P. J. D. Lindan, M. J. Probert et al., "Firstprinciples simulation: ideas, illustrations and the CASTEP code," Journal of Physics Condensed Matter, vol. 14, no. 11, pp. 2717-2744, 2002.

[22] W. E. Pickett, "Density functional in solids: II. excited states," Comments on Solid State Physics, vol. 12, p. 57, 1986.

[23] R. C. Fang, Solid Spectroscopy, Chinese Science Technology University Press, Hefei, China, 2003.

[24] Y. Zhang and W. M. Shen, Basic of Solid Electronics, Zhe Jiang University Press, Hangzhou, China, 2005.

[25] C. M.I. Okoye, "Theoretical study of the electronic structure, chemical bonding and optical properties of $\mathrm{KNbO}_{3}$ in the paraelectric cubic phase," Journal of Physics Condensed Matter, vol. 15, no. 35, pp. 5945-5958, 2003.

[26] R. Asahi, Y. Taga, W. Mannstadt, and A. J. Freeman, "Electronic and optical properties of anatase $\mathrm{TiO}_{2}$," Physical Review $B$, vol. 61, no. 11, pp. 7459-7465, 2000.

[27] Q.-J. Liu, Z.-T. Liu, L.-P. Feng, and H. Tian, "Electronic and optical properties of cubic $\mathrm{SrHfO}_{3}$," Communications in Theoretical Physics, vol. 54, no. 5, pp. 908-912, 2010.

[28] Q.-J. Liu, Z.-T. Liu, Y.-F. Liu, L.-P. Feng, H. Tian, and J. G. Ding, "First-principles study of structural, electronic and optical properties of orthorhombic $\mathrm{SrZrO}_{3}$," Solid State Communications, vol. 150, no. 41-42, pp. 2032-2035, 2010.

[29] Q. Liu, Z. Liu, L. Feng, and B. Xu, "First-principles study of structural, optical and elastic properties of cubic $\mathrm{HfO}_{2}$," Physica B, vol. 404, no. 20, pp. 3614-3619, 2009. 

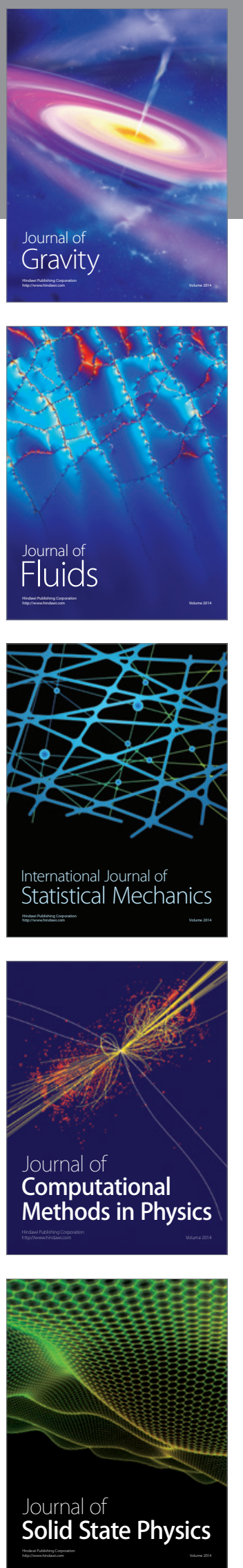
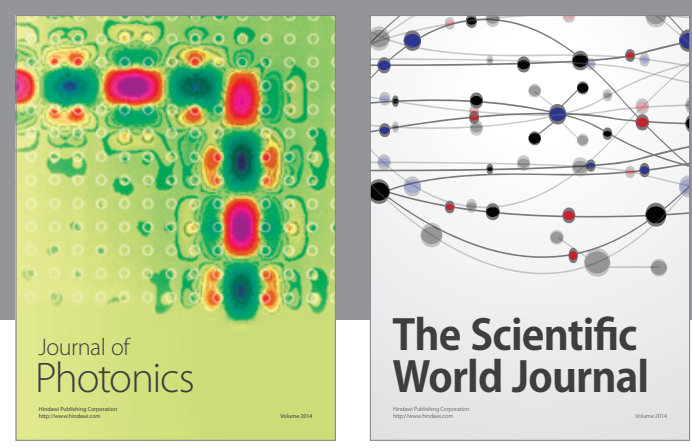

The Scientific World Journal

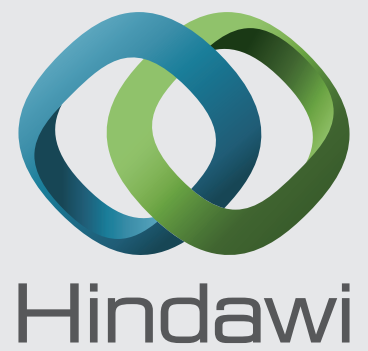

Submit your manuscripts at http://www.hindawi.com
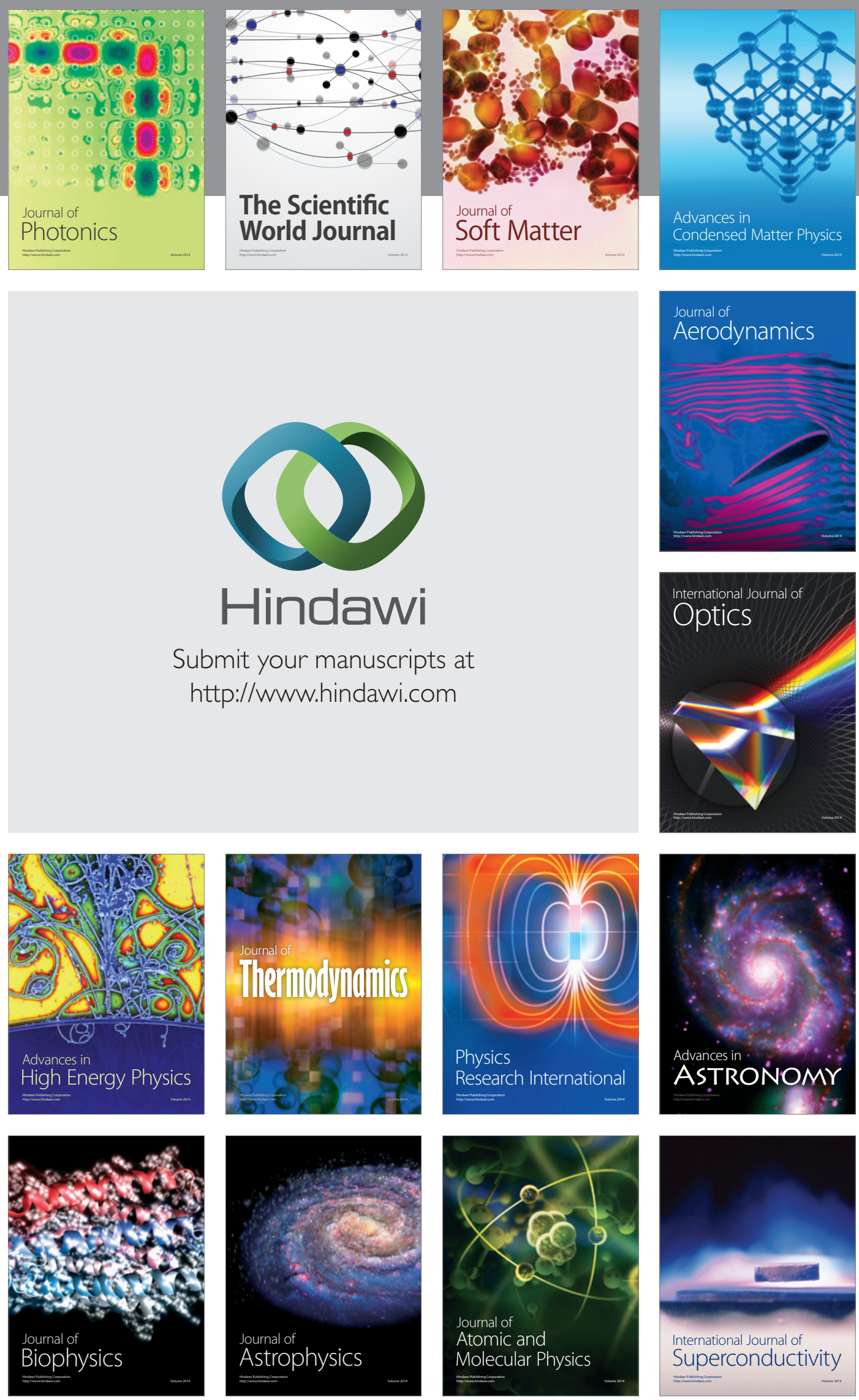
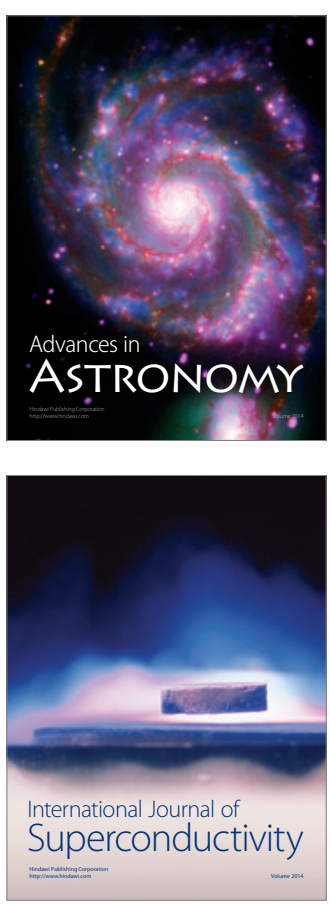